\title{
Editorial
}

\section{Structural Modelling at the Micro-, Meso-, and Nanoscales}

\author{
Angelo Marcello Tarantino, ${ }^{1}$ Julius Kaplunov, ${ }^{2}$ Raimondo Luciano, ${ }^{3}$ Carmelo Majorana, \\ Theodoros C. Rousakis, ${ }^{5}$ and Kasper Willam ${ }^{6}$
}

${ }^{1}$ University of Modena and Reggio Emilia, Modena, Italy

${ }^{2}$ Keele University, Staffordshire, UK

${ }^{3}$ University of Cassino, Cassino, Italy

${ }^{4}$ University of Padova, Padova, Italy

${ }^{5}$ University of Thrace, Komotini, Greece

${ }^{6}$ University of Colorado, Boulder, CO, USA

Correspondence should be addressed to Angelo Marcello Tarantino; angelomarcello.tarantino@unimore.it

Received 24 October 2016; Accepted 24 October 2016; Published 10 January 2017

Copyright (C) 2017 Angelo Marcello Tarantino et al. This is an open access article distributed under the Creative Commons Attribution License, which permits unrestricted use, distribution, and reproduction in any medium, provided the original work is properly cited.

An important target in industry is that of developing new technologies for microelectromechanical systems (MEMS) and nanoelectromechanical systems (NEMS), designed for a multitude of applications in many technical fields. For example, carbon nanotubes are currently considered very prominent materials owing to their excellent electric, thermal, and mechanical properties. These new materials are employed also for purely mechanical purposes. In fact, carbon nanotubes are often used as reinforcement of traditional construction materials, because their application can improve significantly the tensile strength.

Micro-, meso-, and nanostructures need a structural and constitutive modelling consistent with their actual geometrical dimensions. In this regard, it is necessary to develop suitable models that lead to reliable results, since in the literature they are often not available. This special issue is dedicated to theoretical, numerical, and experimental investigations of the behaviour of structures at micro-, meso, and nanoscale. The purpose is to collect and compare the contributions of a research field very timely and of great scientific interest.

The papers in this special issue cover the following topics: (i) static analysis, buckling, and vibration of micro-, meso-, and nanostructures; (ii) nanobeams, nanoshells, and nanoplates; (iii) micro-, meso-, and nanostructural effects; (iv) size effects; (v) microelectromechanical systems (MEMS); (vi) nanoelectromechanical systems (NEMS); (vii) nonlocal theory applied to micro-, meso-, and nanoscales; (viii) nanocomposite materials; (ix) carbon nanotubes; (x) micro- and mesomechanics of concrete; (xi) functionally graded materials; (xii) piezo and magnetic elastic materials; (xiii) homogenization techniques.

The contributions offered by each paper are detailed in the following.

V. Lepov et al. study the problem of brittle fracture of structures at low-temperature conditions connected to damage accumulation and ductile-brittle transition in metals. The internal friction method is applied to revealing the mechanism of dislocation microstructure changes during the low-temperature ductile-brittle transition. It has been shown for the first time that transition is not connected to interatomic interactions but it is stipulated by thermofluctuation on nucleus such as microcracks and by their further growth and coalescence. The proposed mechanism would be used for theoretical and numerical modelling of damage accumulation and fracture in materials.

B. Pomaro considers the radiation damage process in concrete and proposes a unified approach to the practical and predictive assessment of irradiated concrete, which combines both physics and structural mechanics issues. Her paper 
provides a collection of the most distinguished contributions on this topic in the past 50 years up to present, which shows a remarkable renewed interest on the subject.

G. Mazzucco and G. Xotta examine structural elements in concrete exposed to high and rapidly rising temperatures. To reduce the risk of spalling of a concrete material under fire condition, the inclusion of a low dosage of polypropylene fibres in the mix design of concrete is largely recognized. PP fibres in fact evaporate above certain temperatures, thus increasing the porosity and reducing the internal pressure in the material by an increase of the voids connectivity in the cement paste. In their work the contribution of polypropylene fibres on concrete behaviour has been numerically investigated using a coupled hygro-thermal-mechanical finite element formulation at the macro- and mesoscale levels.

G. Mazzucco et al. propose a three-dimensional coupled thermoelectromechanical model for electrical connectors to evaluate local stress and temperature distributions around the contact area of electric connectors. A micromechanical numerical model has been developed by merging together the contact theory approach, which makes use of the socalled roughness parameters obtained from experimental measurements on real contact surfaces, with the topology description of the rough surface via the theory of fractal geometry. In particular, the variation of asperities has been evaluated via the Weierstrass-Mandelbrot function. In this way the micromechanical model allowed for an upgraded contact algorithm in terms of effective contact area and thermal and electrical contact conductivities. A comparison between numerical and analytical results shows that the adopted procedure is suitable to simulate the transient thermoelectromechanical response of electric connectors.

L. Feo and R. Penna employ the Eringen elastic constitutive relation to assess small-scale effects in nanobeams. Structural behaviour is studied for functionally graded materials in the cross-sectional plane and torsional loading conditions. Torsional rotations and equilibrated moments are evaluated by solving a first-order differential equation of elastic equilibrium with boundary conditions of kinematic-type. Benchmarks examples are briefly discussed, thus enlightening the effectiveness of the proposed methodology.

P. Di Maida and G. Bianchi perform a pseudospectral approximation to solve the problem of pull-in instability in a cantilever microswitch. A numerical comparison is presented between a pseudospectral and a finite element (FE) approximation of the problem. It is shown that the pseudospectral method appears more effective in accurately approximating the behaviour of the cantilever near its tip. This fact is crucial to capturing the threshold voltage on the verge of pull-in. Conversely, the FE approximation presents rapid successions of attracting/repulsing regions along the cantilever, which are not restricted to the near pull-in regime.

M. Diaco analyses orthotropic Saint-Venant beams under torsion, for which a skillful solution method has been recently proposed by Ecsedi considering a class of inhomogeneous beams with shear moduli defined in terms of Prandtl's stress function of corresponding homogeneous beams. Using this approach, orthotropic functionally graded beams with shear moduli tensors defined in terms of the stress function are studied. A result of invariance on twist centre is also achieved. Examples of functionally graded elliptic crosssections of orthotropic beams are developed, thus detecting new benchmarks for computational mechanics.

V. P. Berardi presents a micromechanical procedure to evaluate the initiation of damage and failure of masonry with in-plane loads. Masonry material is viewed as a composite with periodic microstructure and, therefore, a unit cell with suitable boundary conditions is assumed as a representative volume element of the masonry. The finite element method is used to determine the average stress on the unit cell corresponding to a given average strain prescribed on the unit cell. Finally, critical curves representing the initiation of damage and failure in both clay brick masonry and adobe masonry are provided.

P. Di Maida and F. O. Falope investigate the contact problem of an Euler-Bernoulli nanobeam of finite length bonded to a homogeneous elastic half plane. The analysis is performed under plane strain condition. Owing to the bending stiffness of the beam, shear and peeling stresses arise at the interface between the beam and the substrate within the contact region. The investigation allows evaluating the role played by the Poisson ratio of the half plane (and, in turn, its compressibility) on the beam-substrate mechanical interaction. Different symmetric as well as skew-symmetric loading conditions for the beam are considered, with particular emphasis on concentrated transversal and horizontal forces and couples acting at its edges. It is found that the Poisson ratio of the half plane affects the behaviour of the interfacial stress field, particularly at the beam edges, where the shear and peel stresses are singular.

F. O. Falope and E. Radi study the mechanical behaviour of thin films bonded to a homogeneous elastic orthotropic half plane under plane strain condition. Both the film and semi-infinite substrate display linear elastic orthotropic behaviour. By assuming perfect adhesion between film and half plane together with membrane behaviour of the film, the compatibility condition between the coating and substrate leads to a singular integral equation with Cauchy kernel. Such an equation is straightforwardly solved by expanding the unknown interfacial stress in series of Chebyshev polynomials displaying square-root singularity at the film edges. This approach allows handling the singular behaviour of the shear stress and, in turn, reducing the problem to a linear algebraic system of infinite terms. Results are found for two loading cases, with particular reference to concentrated axial forces acting at the edges of the film. The corresponding mode II stress intensity factor has been assessed, thus providing the stress concentrations at both ends of the covering. Possible applications of the results here obtained range from MEMS, NEMS, and solar silicon cell for energy harvesting to welded joint and building foundation.

A. Sorzia carries out creep and relaxation tensile tests until breakage of polypropylene fibres. Creep and stress relaxation curves are obtained and fit by a model adopting a fraction-exponential kernel in the viscoelastic operator. Taking into account the fact that the addition of polypropylene fibres greatly improves the tensile strength of composite materials with concrete matrix, the proposed analytical 
model is used for simulating the mechanical behaviour of composite materials with embedded viscoelastic fibres.

L. Feo and R. Penna evaluate size effects in functionally graded elastic nanobeams by making recourse to the nonlocal continuum mechanics. The Bernoulli-Euler kinematic assumption and the Eringen nonlocal constitutive law are assumed in the formulation of the elastic equilibrium problem. An innovative methodology, characterized by a lowering in the order of governing differential equation, is adopted to solve the boundary value problem of a nanobeam under flexure. Unlike standard treatments, a second-order differential equation of nonlocal equilibrium elastic is integrated in terms of transverse displacements and equilibrated bending moments. Benchmark examples are developed, thus providing the nonlocality effect in nanocantilever and clamped-simply supported nanobeams for selected values of the Eringen scale parameter.

A. Sorzia applies the Floquet theory of periodic coefficient second-order ODEs to an elastic waveguide. The waveguide is modelled as a uniform elastic string periodically supported by a discontinuous Winkler elastic foundation and, as a result, a Hill equation is found. The fundamental solutions, the stability regions, and the dispersion curves are determined. An asymptotic approximation to the dispersion curve is also provided. It is further shown that the end points of the band gap structure correspond to periodic and semiperiodic solutions of the Hill equation.

The scope of the works presented in this special issue offers a real insight into the progress made across a wide range of theoretical and technical topics related to structural modelling at the micro-, meso-, and nanoscales. We hope that this special issue will stimulate further research interests.

\title{
Acknowledgments
}

On behalf of the guest editors, we would like to acknowledge all the editorial members, contributed authors, and dedicated referees for their invaluable time, great contributions, and assistance to this volume. Without such efforts we would not be able to accomplish this very interesting and informative special issue.

\author{
Angelo Marcello Tarantino \\ Julius Kaplunov \\ Raimondo Luciano \\ Carmelo Majorana \\ Theodoros C. Rousakis \\ Kasper Willam
}




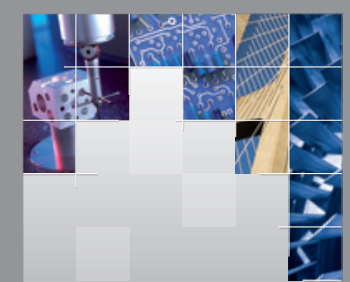

\section{Enfincering}
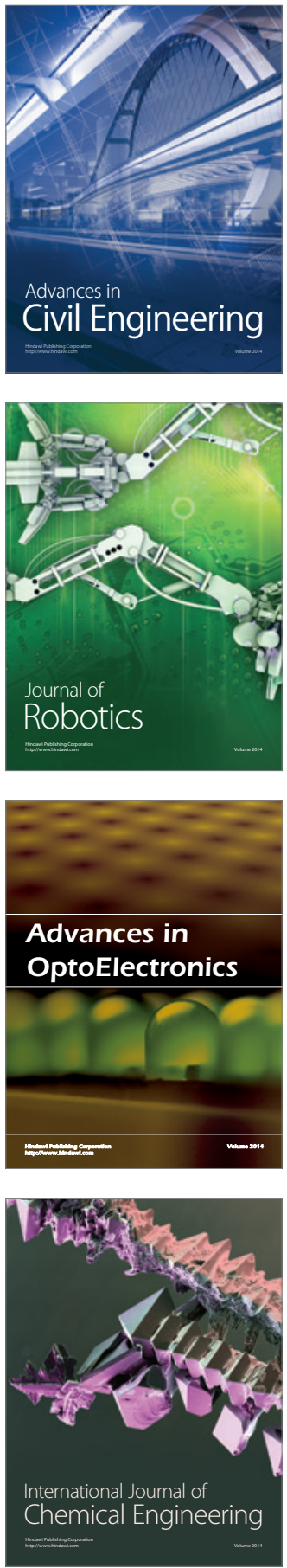

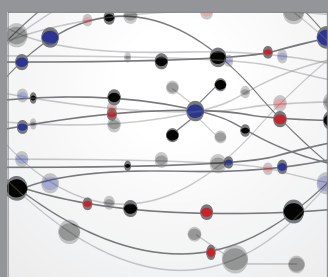

The Scientific World Journal

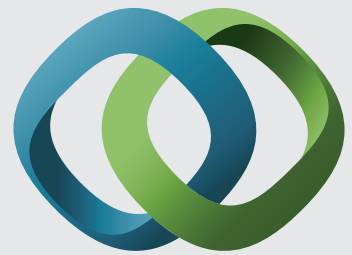

\section{Hindawi}

Submit your manuscripts at

https://www.hindawi.com
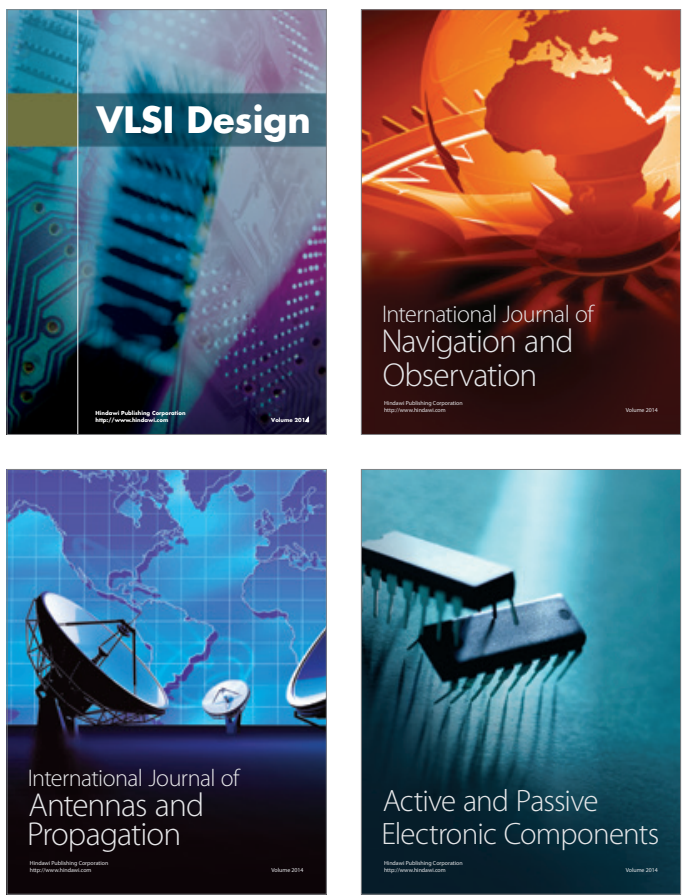
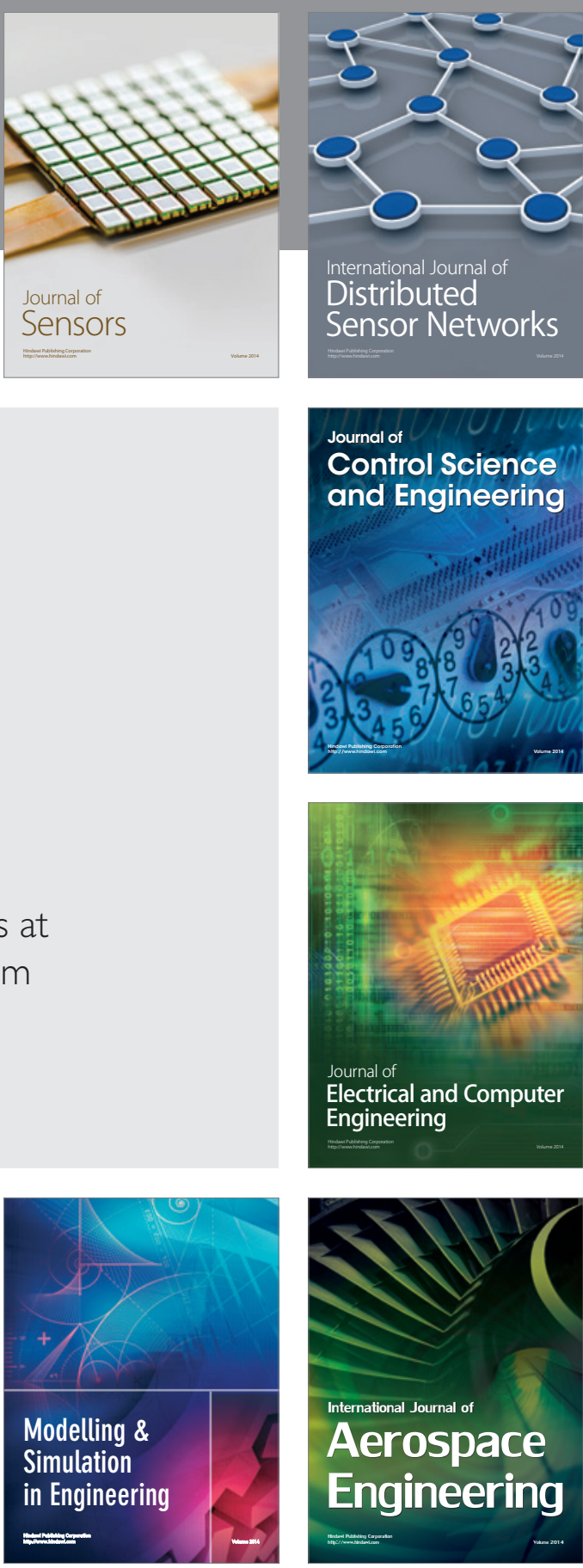

International Journal of

Distributed

Sensor Networks

$-$

Joumal of

Control Science

and Engineering
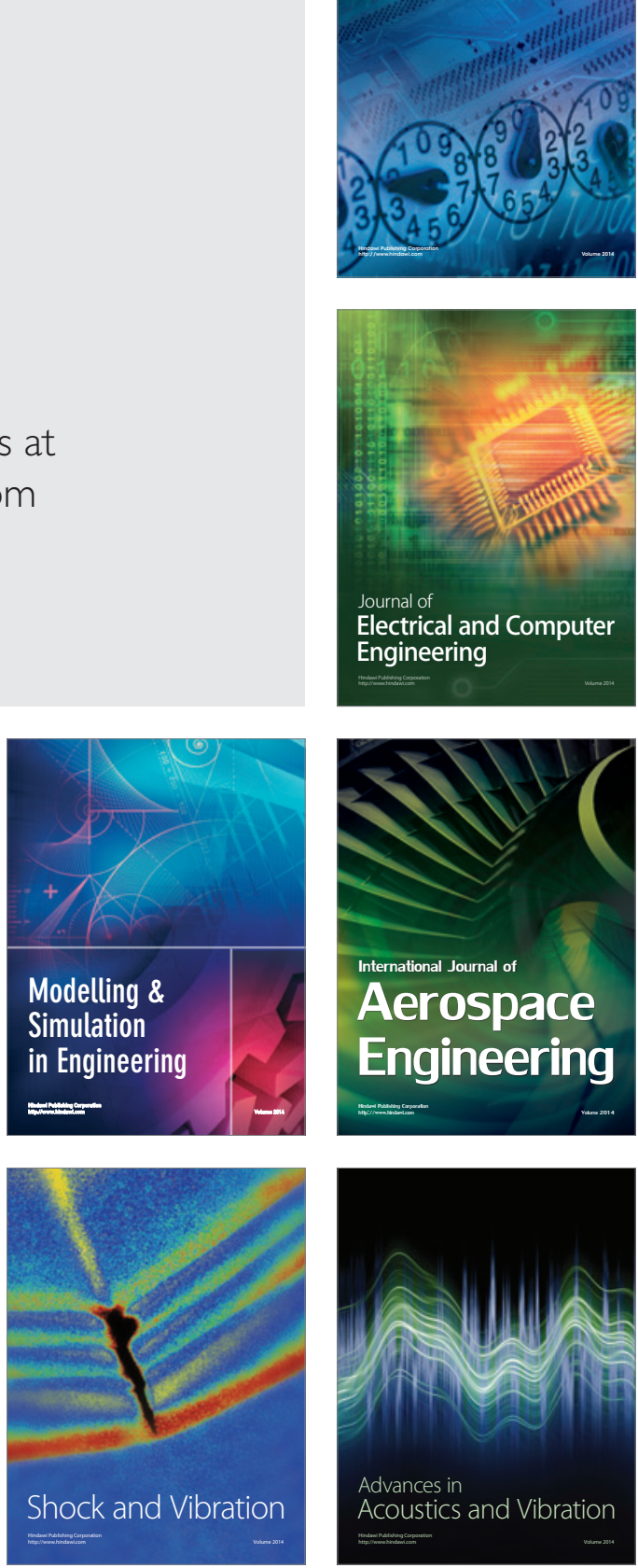